\title{
Modélisation des turbidités en Bretagne Sud et validation par des mesures in-situ
}

\author{
Caroline Tessier ${ }^{1}$, Pierre Le Hir ${ }^{1}$, Franck Dumas ${ }^{1}$ et F. Jourdin ${ }^{2}$ \\ ${ }^{1}$ IFREMER DYNECO/PHYSED Centre de Brest \\ BP 70 - 29280 Plouzané \\ ${ }^{2}$ EPSHOM-CMO/REC - BP 30316 - 29603 Brest
}

\begin{abstract}
Résumé
Un modèle hydro-sédimentaire de la Bretagne Sud est calibré avec des mesures $\mathrm{ADCP}$, qui permettent d'estimer les forçages hydrodynamiques et les concentrations de matière en suspension. L'exercice de comparaison entre concentrations observée et simulée révèle la forte dépendance de la turbidité à de nombreux processus sédimentaires et physiques, fortement couplés entre eux.
\end{abstract}

\begin{abstract}
A hydrodynamic and sediment model of South Brittany is calibrated with ADCP measurements, which allow to estimate hydrodynamic forcing and concentration of suspended particulate matter. The exercise of comparison between observed and simulated concentrations reveals that turbidity is highly dependant on numerous strongly coupled sedimentary and physical processes.
\end{abstract}

Mots-clés: turbidité, modélisation, rétrodiffusion acoustique, ADCP, vagues.

\section{Introduction}

La modélisation des turbidités en Bretagne Sud a été entreprise afin de mieux comprendre et représenter la dynamique des sédiments fins dans la zone, mais aussi de mieux prévoir les développements d'espèces phytoplanctoniques, très sensibles à la pénétration de la lumière. L'objectif est ici d'évaluer les performances d'un modèle hydro-sédimentaire avec un ensemble de mesures acquises en station fixe avec un profileur acoustique de courant (ADCP) : courant, houle et concentration massique obtenue à partir de l'intensité rétrodiffusée. Après une description du modèle et des mesures ADCP, il s'agit de discuter les résultats et les sensibilités sur l'estimation des turbidités, évaluées ici en terme de concentration massique.

\section{Modélisation du transport des particules fines en Bretagne sud}

\subsection{Courants}

Le modèle aux différences finies MARS-3D (Lazure et Dumas, 2006), développé à l'IFREMER, a été configuré avec des mailles variables (700-2000 m) et huit niveaux sigma (épaisseurs des couches variables en fonction de la hauteur d'eau), 
en simulation réaliste sur une zone allant de la pointe de Penmarc'h à l'île d'Yeu. Il intègre le forçage par la marée, le vent (ARPEGE Météo-France) et les effets de densité générés par les apports des fleuves. La marée est imposée à la limite ouverte, calculée avec un modèle 2D de plus grande emprise. Les flux solaires sont modélisés selon Luyten et al. (1992). De plus, un appel aux climatologies de Reynault et al. (1998) est fait en température et salinité à la limite ouverte. La longueur de rugosité du fond considérée pour les courants intègre d'éventuels effets des rides : elle est choisie égale à $1 \mathrm{~mm}$ sur toute la zone. La fermeture turbulente et sa paramétrisation sont discutées plus loin.

\subsection{Houles}

L'effet des houles et de la mer de vent est pris en compte à partir de simulations instationnaires du modèle de propagation SWAN (Booij et al., 1999), configuré sur la même zone et le même maillage irrégulier. Les variations de hauteur d'eau, la génération/dissipation par le vent, la friction sur le fond, les interactions non linéaires et la réfraction par les courants sont prises en compte. Ce modèle est forcé le long de sa limite ouverte par un ensemble de spectres directionnels, fournis par Fabrice Ardhuin (SHOM) à partir de simulations de WAVEWATCHIII à 0.1 degré de résolution (forcé par les vents ARPEGE et emboîté dans un modèle de l'Atlantique Nord).

\subsection{Sédiment}

Le modèle MARS intègre les modules d'érosion, dépôt et tassement de sédiments multicouches du modèle SiAM-3D (Cugier et Le Hir, 2002). Le tassement n'est pas considéré ici. Un fond sédimentaire quasi-réaliste est pris en compte dans le modèle, avec trois classes de particules (sable moyen, sable fin et vase). Les graviers et cailloutis ne sont pas considérés. La dynamique des sables étant limitée à la couche de fond, on s'intéresse ici aux particules fines uniquement, dont la vitesse de chute est paramétrée en fonction de la concentration.

\section{Mesures in-situ ADCP}

En collaboration avec le SHOM, un mouillage expérimental a été mis en place dans le Mor-Bras en octobre 2004 (position sur la figure 5), avec un ADCP Broadband $1200 \mathrm{kHz}$ (RDI) posé au fond pendant dix jours : les courants et l'intensité rétrodiffusée du signal sont échantillonnés tous les demi-mètres sur la colonne d'eau, à partir de $1,60 \mathrm{~m} \mathrm{du}$ fond, toutes les $10 \mathrm{mn}$. L'appareil a également mesuré les spectres de vagues chaque heure (calculs avec le logiciel WavesMon, RDI). Un courantomètre ADCP de fréquence $300 \mathrm{kHz}$ avait également été mouillé à proximité : le parfait accord sur les vitesses a permis une validation a posteriori de cette mesure.

\subsection{Estimation des contraintes}


Le remaniement des sédiments fait appel à une estimation fine des contraintes de peau générées par les vagues et les courants. La contrainte liée au courant de marée est calculée à partir de la vitesse près du fond $u(z)$ et d'une rugosité de peau $z_{0}$, selon $: \tau_{c}=\rho_{0} u_{*}{ }^{2}$, avec $u^{*}=\kappa \cdot u(z) / \ln \left(z / z_{0}\right), \rho_{0}$ étant la masse volumique de l'eau, $z$ la hauteur par rapport au fond et $\kappa$ la constante de von Karman.

La contrainte liée aux houles est paramétrisée à l'aide du facteur de frottement $f_{w}$ : $\tau_{w}=0,5 f_{w} \rho_{0} U_{b o t}{ }^{2}$, où $U_{b o t}$ est la vitesse orbitale au fond. $f_{w}$ est pris comme le maximum des deux formulations suivantes, qui correspondent aux régimes laminaire $\left(f w_{L}\right)$ ou turbulent rugueux $\left(f w_{R T}\right)$ :

- $\quad f w_{L}=2 R w^{-0,5}$, avec le nombre de Reynolds $R w=U_{b o t} A / v$, où $A$ est l'excursion orbitale près du fond et $v$ est la viscosité du fluide,

- $\quad f w_{R T}=1,39\left(A / z_{0}\right)^{-0,52}$ (in Soulsby, 1997).

$U_{b o t}$ et $A$ sont calculés à partir des spectres :

$$
U_{b o t}{ }^{2}=2 U_{r m s}{ }^{2}=2 \sum_{i} \frac{4 \pi^{2} f_{i}^{2} h_{i} d f_{i}}{\sinh ^{2}\left(2 \pi H / \lambda_{i}\right)} \text { et } A_{\text {spec }}{ }^{2}=2 \sum_{i}\left(h_{i} d f_{i} / \sinh ^{2}\left(2 \pi H / \lambda_{i}\right)\right) \text {, avec }
$$

$h_{i}$ la composante $\mathrm{i}$ du spectre de houle $\left(\mathrm{m}^{2} / \mathrm{Hz}\right), \lambda_{\mathrm{i}}$ la longueur d'onde associée à la fréquence i du spectre et $H$ la hauteur d'eau.

Devant l'incertitude sur la valeur de la rugosité du fond $z_{0}$, les contraintes sont estimées pour deux valeurs différentes, $z_{01}=0,1 \mathrm{~mm}$ et $z_{02}=0,033 \mathrm{~mm}$, les fonds de la zone étudiée étant composés de vase à plus de $80 \%$. Les mesures ADCP donnent : $R w<710^{-4}$ et $A /\left(30 z_{01}\right)=50-300$. L'écoulement est en régime de transition, avec $0,01<f w_{L}<0,05$ et $f w_{R T} \geq f w_{L}$ pour $z_{01}$ mais $f w_{R T} \leq f w_{L}$ pour $z_{02}$. Pour les événements de houle observés durant cette période, avec des hauteurs significatives atteignant deux mètres, les contraintes liées aux houles sont ainsi de 0,6 ou $0,3 \mathrm{~Pa}$ selon que l'on considère une rugosité $z_{01}$ ou $z_{02}$. L'interaction houlecourant, pour le calcul de la contrainte sur le fond, est prise en compte par la formulation de Soulsby (1997). La contrainte maximale est indiquée sur la figure 1 pour les deux valeurs de rugosités; l'écart est de $40 \%$ lors des pics de houle.

Pour la modélisation, nous avons retenu le paramètre $z_{01}$, plus représentatif des rugosités physiques de peau des sédiments naturels. Le facteur de frottement appliqué est $f w_{R T}$, dans lequel $A$ est estimé avec la période moyenne (plus "représentative" pour $f w$ que la période de pic, d'après les mesures ADCP). Par contre, $U_{b o t}$ est calculé spectralement par SWAN.

\subsection{Obtention de la concentration massique à partir du signal rétrodiffusé}

Le signal acoustique de l'ADCP est rétrodiffusé par les particules présentes dans la colonne d'eau, et dépend donc de la concentration. En corrigeant des pertes de transmission de l'onde au cours de sa propagation, qui dépendent des conditions hydrologiques, on obtient un indice de rétrodiffusion des particules dans l'eau qui varie avec leur concentration et leur nature. Cet indice, normalisé par unité de volume rétrodiffusant, peut être calibré directement en concentration massique 
avec des mesures indépendantes, pesées MES et turbidité optique (Tessier et al., 2006). Les calibrations obtenues ici sont présentées sur la figure 2 . Les valeurs de concentration massique obtenues à partir des signaux ADCP (figure 1) fluctuent de 10 à $50 \mathrm{mg} / \mathrm{l}$ à trois mètres du fond, en fonction des houles et de la marée, mais aussi en fonction des coups de vent, qui induisent des mélanges importants de la colonne d'eau.
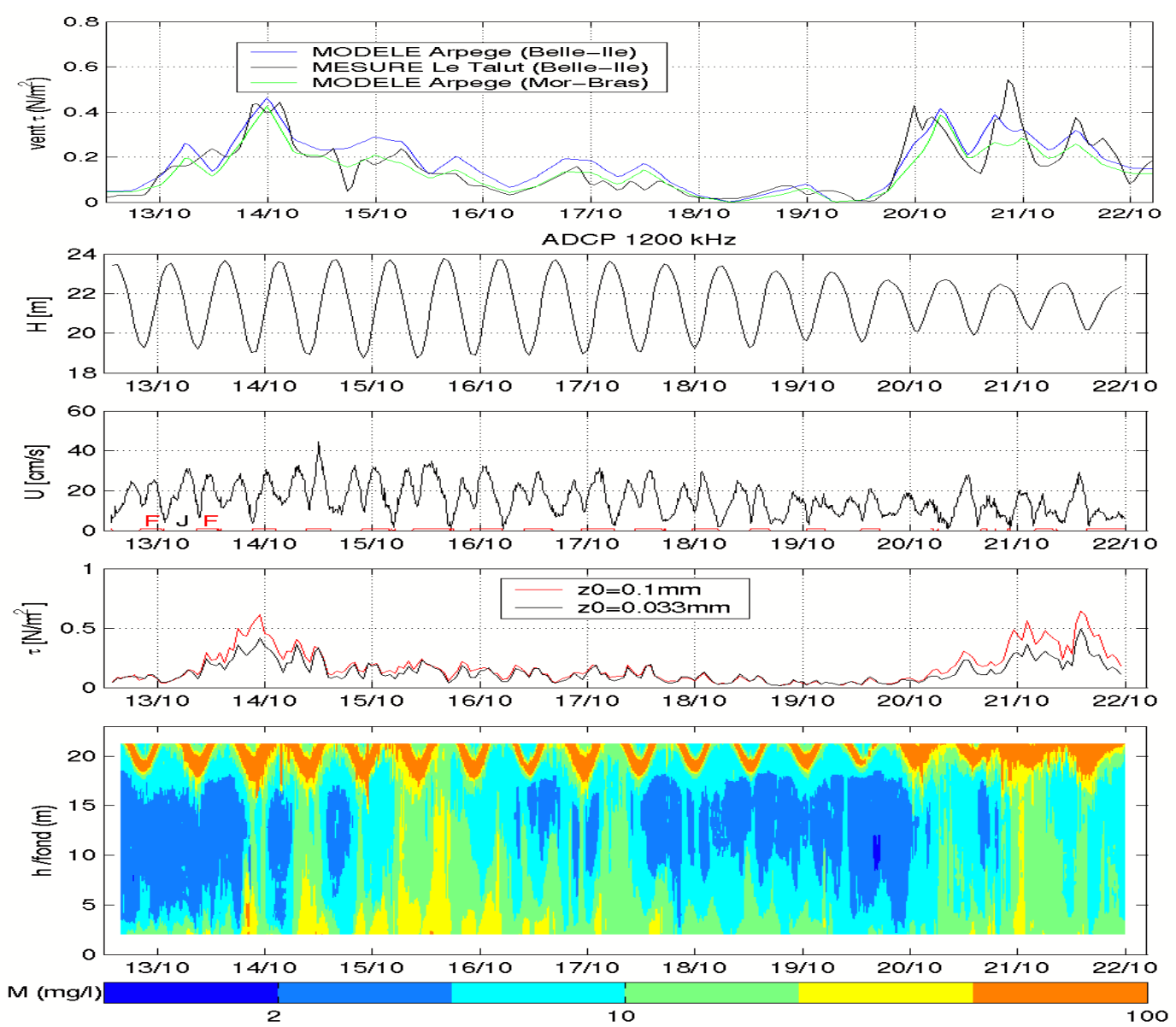

Figure 1: Tension de vent du modèle Arpège à Belle-Ile et dans le Mor-Bras, et mesuré à Belle-Ile ; Mesures de l'ADCP $1200 \mathrm{kHz}$ en octobre 2004 : hauteur d'eau $(\mathrm{m})$, intensité du courant à $3 \mathrm{~m}$ du fond $(\mathrm{cm} / \mathrm{s})$ (Flot en rouge), contrainte houle + courant $\left(\mathrm{N} / \mathrm{m}^{2}\right)$ pour deux valeurs de $\mathrm{z}_{0}$, concentration massique $(\mathrm{mg} / \mathrm{L})$ en fonction de la hauteur par rapport au fond (l'écho de la surface libre apparaît en orange).
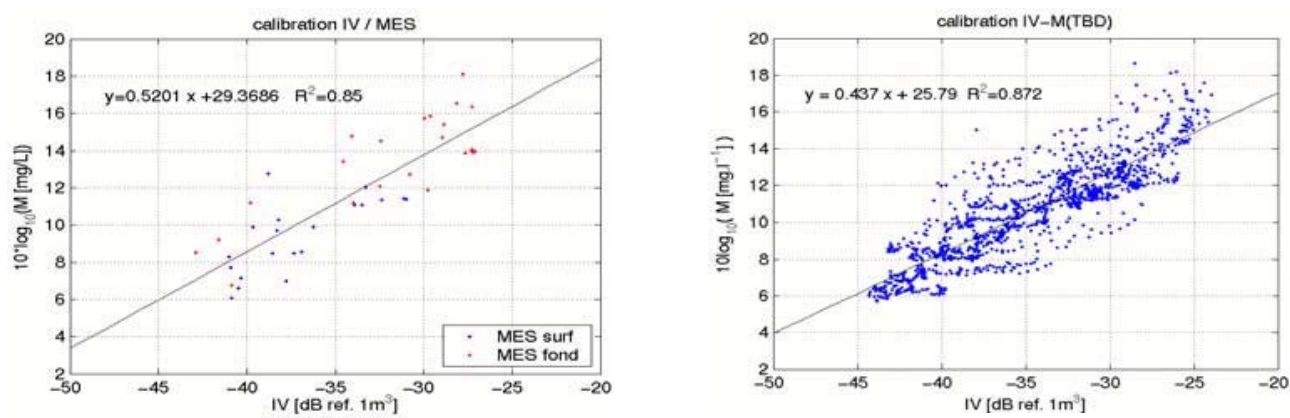

Figure 2 : Calibration de l'indice de rétrodiffusion IV avec les pesées MES (gauche) et avec le turbidimètre optique TBD calibré (droite) 


\section{Résultats du modèle et sensibilités}

\subsection{Houles et contraintes globales}

Les résultats du modèle SWAN sont comparés aux mesures de houles en trois points : à l'Ile d'Yeu (bouée CETMEF YEU), à l'embouchure de la Loire (bouée CETMEF ST-NAZAIRE) et dans le Mor-Bras (ADCP). Les positions des points sont indiqués sur la figure 4. Les variations temporelles du forçage sont bien reproduites aux trois points. La figure 3 montre les comparaisons à l'embouchure de la Loire et dans le Mor-Bras. La période moyenne de la bouée est plus grande du fait de sa fréquence maximale de $0.5 \mathrm{~Hz}$. La période moyenne calculée par le modèle prend donc en compte des hautes fréquences que ne voit pas la bouée. Le modèle montre une légère sensibilité à la paramétrisation de la génération/dissipation par le vent et une plus forte à la friction sur le fond, ellemême contrôlée par les macro-rugosités du fond (paramètre $k_{N}$ de rugosité de Nikuradse). Pour mieux reproduire l'atténuation des vagues à la côte, $\mathrm{k}_{\mathrm{N}}$ est pris égal à $0,3 \mathrm{~m}$, au lieu de la valeur $0,05 \mathrm{~m}$ par défaut. Les hauteurs significatives simulées restent parfois trop fortes (14-16/10/2004), mais comme les résultats sont corrects à St-Nazaire, l'imprécision dans le Mor-Bras peut être imputée à un effet du vent local ou à une atténuation par la liquéfaction des vases dans la zone. Cependant, les tentatives de spatialisation de la rugosité du fond n'ont pas été concluantes.

La figure 4 illustre une distribution des houles en situation de tempête. Le rôle d'abri du Mor Bras par les îles et Quiberon est clair, mais la baie de Vilaine reste exposée et les vitesses orbitales (et donc le frottement sur le fond) n'y sont pas négligeables. Dans ce type de zone peu profonde au fond sédimentaire facilement remobilisable, l'action des houles joue un rôle important dans la génération des turbidités côtières. Les courants de marée y sont faibles mais sont responsables du mélange vertical et du transport des particules remises en suspension par les houles.
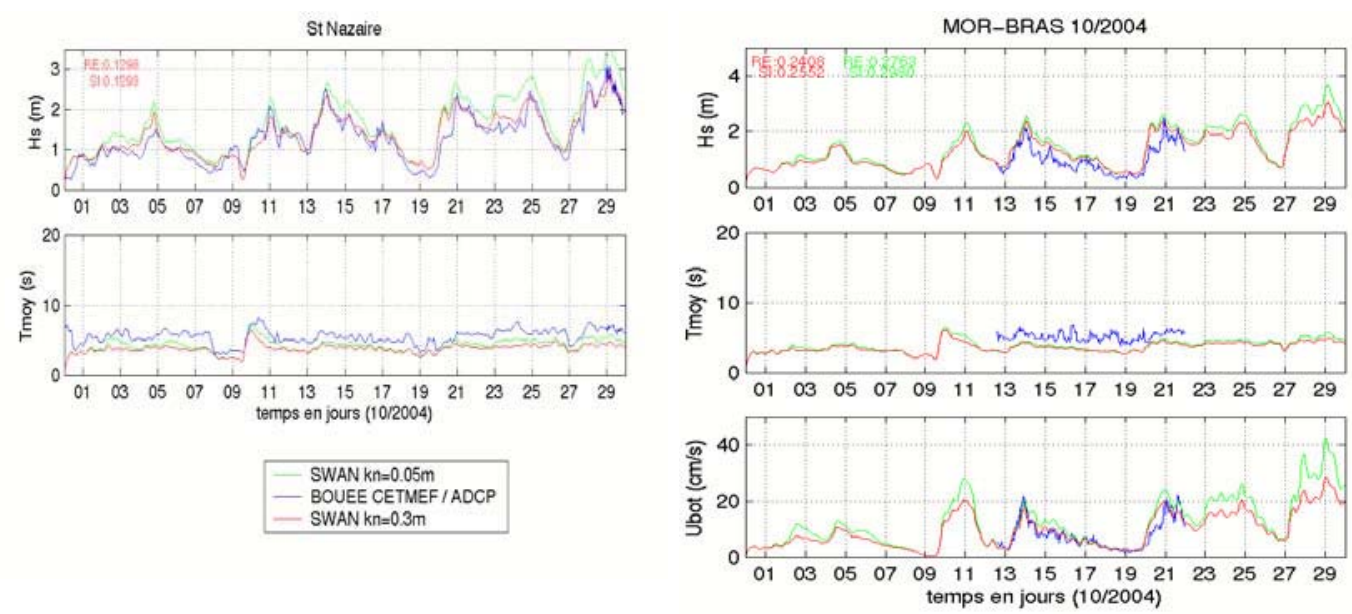

Figure 3: hauteur significative $(\mathrm{m})$, période moyenne (s) et vitesse orbitale au fond $(\mathrm{cm} / \mathrm{s})$ dans le Mor-Bras (droite) et à l'embouchure de la Loire (gauche): mesures (bleu) [Bouée CETMEF de St-Nazaire / ADCP dans le Mor-Bras] et simulations $\mathrm{SWAN}$ pour $\mathrm{k}_{\mathrm{N}}=0,3 \mathrm{~m}$ (rouge) et $\mathrm{k}_{\mathrm{N}}=0,05 \mathrm{~m}$ (vert). 

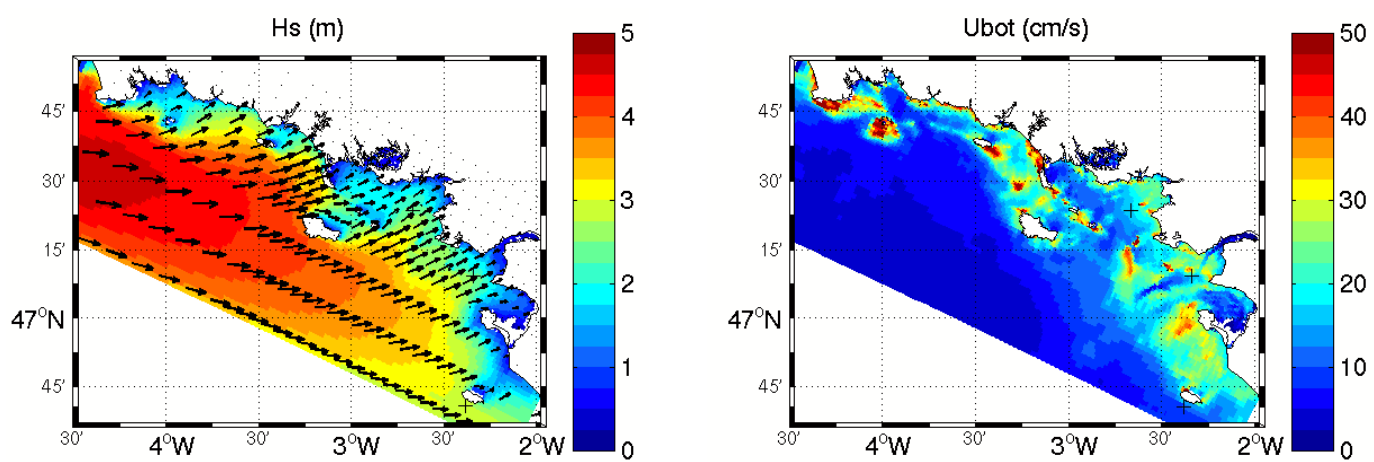

Figure 4: Simulation SWAN $\mathrm{k}_{\mathrm{N}}=0,3 \mathrm{~m}$, sortie du 14/10/2004 00:00:00. Hauteur significative (m) et incidence des houles (gauche), vitesse orbitale au fond $(\mathrm{cm} / \mathrm{s})$ (droite). Les trois points de mesure sont indiqués par des croix noires.

\subsection{Turbidités}

La simulation de référence pour les turbidités a été faite avec le paramétrage suivant :

- Erosion: $E=E_{0}\left(\tau / \tau_{c e^{-1}}\right)$, avec $\tau_{c e}=0,25 \mathrm{~N} / \mathrm{m}^{2}$ et $E_{0}=1,310^{-6} \mathrm{~kg} / \mathrm{m}^{2} / \mathrm{s}$,

- Dépôt: $D=W s C\left(1-\tau / \tau_{c d}\right)$, avec $\tau_{c d}=\tau_{c e}$ et $W_{S}$ variant de $0.1 \mathrm{~mm} / \mathrm{s}$ (faibles concentrations) à $6 \mathrm{~mm} / \mathrm{s}$ (pour $C=1 \mathrm{~g} / \mathrm{l})$ (Le Hir et al., 2001),

- vent du modèle ARPEGE, variable spatialement,

- fermeture turbulente de Luyten et al. (1996), qui résout l'équation de transport de l'énergie cinétique turbulente (ECT) avec un taux de dissipation fonction d'une longueur de mélange paramétrée.

Les résultats de la simulation de référence sont illustrés par la figure 5 (distribution surface/fond des turbidités après tempête) et la figure 6 (évolution du profil de turbidité au point de mouillage de l'ADCP, donc directement comparable à la figure 1). La figure 6 met en évidence une remise en suspension localisée en baie de Vilaine, dont l'extension sud est proche du point de mesure ADCP. On comprend ainsi qu'une modulation des paramètres ou forçages du modèle est susceptible d'influencer fortement sa capacité à reproduire les mesures. C'est ce qui a été vérifié par une série de simulations non illustrées ici.
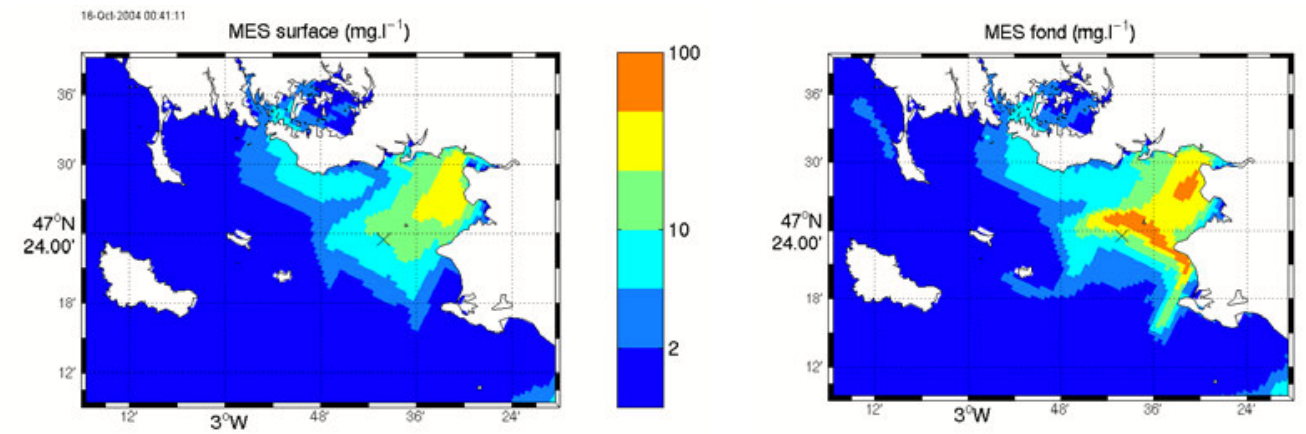

Figure 5: MODELE: Concentrations massiques (mg/l) en surface (droite) et au fond (gauche) le 16/10/2004 (fermeture turbulente de Luyten, vent Arpege). La croix noire situe le point de mesure ADCP.

Naturellement, les vagues sont l'agent principal des remises en suspension, mais les simulations révèlent que les turbidités calculées à l'emplacement de l'ADCP 
seraient fortement influencées par un transport advectif. La figure 6 montre en effet un retard des concentrations par rapport aux contraintes. Cette contribution dépend non seulement de l'importance des remises en suspension dans les secteurs moins profonds du nord de la baie, encore atteints par les vagues, mais aussi du schéma de circulation 3D dans toute la baie. En effet, le courant de marée y est assez faible en dehors des chenaux, et les vents contribuent pour une large part à l'hydrodynamique (cela est même visible en remplaçant le forçage "ARPEGE" par un champ de vent uniforme reconstitué à partir des mesures au sémaphore du Talut). La circulation générée par le vent est elle-même dépendante des conditions de stratification qui sont liées aux apports fluviaux (la Vilaine, mais aussi le panache de la Loire) : dans le modèle 3D, tout cela est contrôlé par la fermeture turbulente que l'état de l'Art ne permet pas de formuler de façon indiscutable. Les résultats au point fixe avec la fermeture turbulente de Gaspar (1990), sur la figure 6, montrent des différences liées à la grande proximité du panache turbide, mais qui restent malgré tout d'un bon ordre de grandeur, par rapport aux mesures.

Une ambiguïté demeure également sur les flux de remise en suspension (localement, et dans le reste de la baie), le calcul de contrainte de cisaillement étant lui-même sensible au paramètre de rugosité mal connu. La figure 6 montre d'autre part que les variations de contraintes calculées et déduites de la mesure ne sont pas parfaitement corrélées, révélant ici un léger désaccord sur la propagation des vagues (ou leur dépendance du vent local).

Cet exercice de comparaison entre turbidités observée et simulée révèle la forte dépendance de la turbidité à de nombreux processus sédimentaires et physiques, ces derniers étant fortement couplés entre eux.
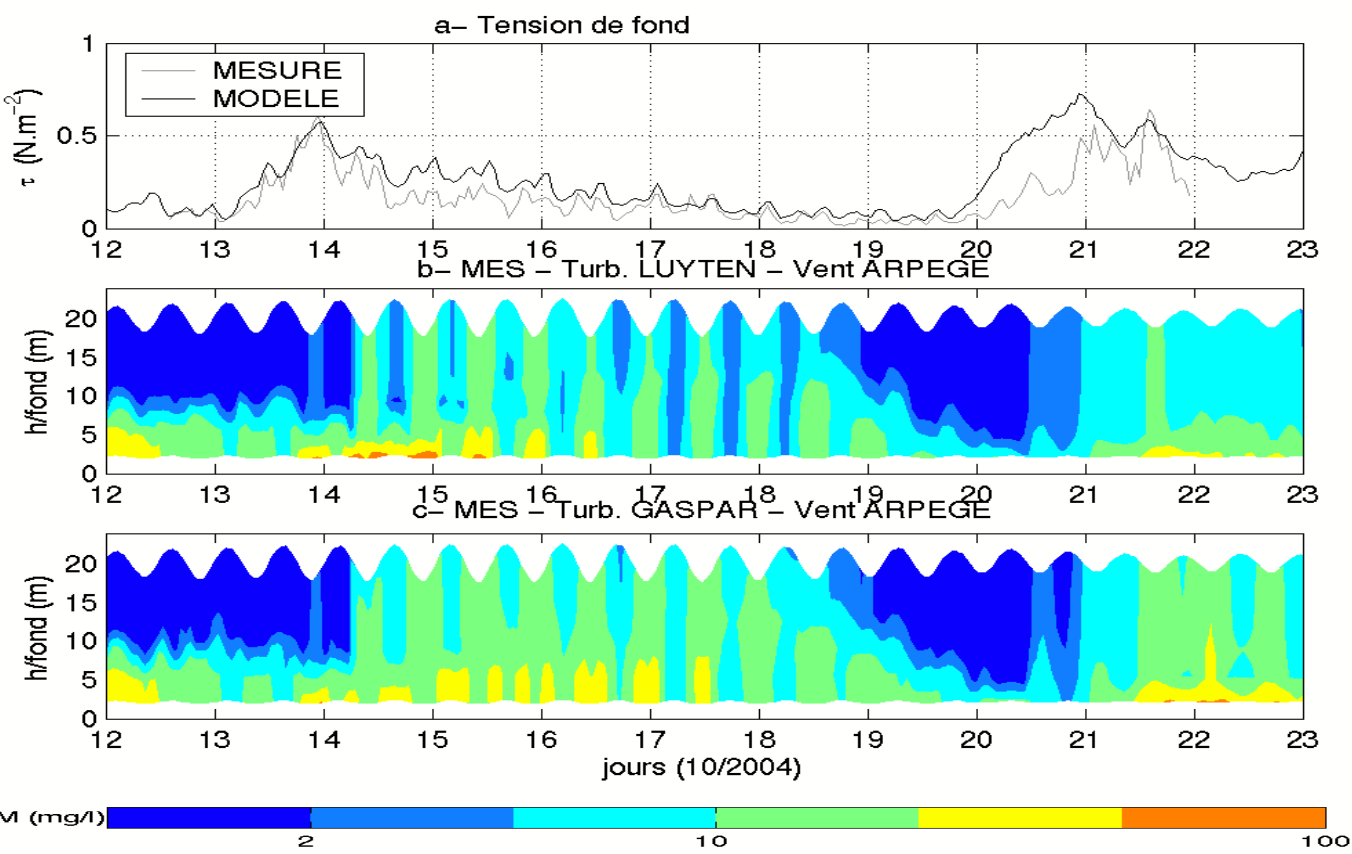

Figure 6: Résultats du modèle au point ADCP (Mor-Bras) en fonction du temps : (a) contrainte houle + courant ; (b) et (c) concentration massique en fonction de la hauteur par rapport au fond. (b) Turbulence de Luyten et al. (1996) et vent Arpege (c) Turbulence de Gaspar (1990) et vent Arpege. 


\section{$\underline{\text { 5. Conclusion }}$}

Les mesures ADCP acquises en continu pendant une dizaine de jours dans le MorBras ont permis d'évaluer la variabilité des forçages hydrodynamiques et d'estimer les concentrations massiques dans la colonne d'eau. Une calibration du modèle hydro-sédimentaire de la Bretagne Sud a alors été faite, avec une paramétrisation simple des phénomènes d'érosion et de dépôt des particules fines. $\mathrm{Du}$ fait de la complexité du site étudié, exposé aux houles du large et aux panaches des fleuves (Loire, Vilaine), l'étude a montré la sensibilité des turbidités au vent, à la structure thermohaline, et à la fermeture turbulente, qui contribuent ensemble à modifier le transport et le mélange, et donc le flux de remise en suspension.

\section{Remerciements}

Le mouillage et les mesures en mer ont été réalisées avec le SHOM et la MHA (campagne OPTIC-PCAF 2004, à bord du "La Pérouse"). Nous remercions aussi le CETMEF pour les données des houlographes, et F. Ardhuin pour les spectres de houles WAVEWATCH-III.

\section{Références}

Booij, N., Ris, R.C., et Holthuijsen, L.H. (1999), A third-generation wave model for coastal regions. 1. Model description and validation. Journal of Geophysical Research, 104 (C4), 7649-7666.

Cugier, P. et Le Hir, P. (2002), Development of a 3D Hydrodynamic model for coastal ecosystem modelling. Application to the plume of the Seine River (France). Estuarine, Coastal and Shelf Science, 55, 673-695.

Gaspar, J.P., Grégoris, Y., et Lefevre, J.M. (1990), A simple eddy kinetic energy model for simulations of oceanic vertical mixing : Tests at station Papa and long-term upper ocean study site. J. Geophys. Res., 95 (C9), 16179-16193.

Lazure, P. et Dumas, F. (2006), A 3D hydrodynamical model for applications at the region scale (MARS-3D) : Application to the bay of Biscay. Ocean Modelling, submitted.

Le Hir, P., Ficht, A., Silva Jacinto, R., Lesueur, P., Dupont, J.-P., Lafite, R., Brenon, I., Thouvenin, B., et Cugier, P. (2001), Fine sediment transport and accumulations at the mouth of the Seine Estuary (France). Estuaries, 24 (6B), $950-963$.

Luyten, P., Deleersnijder, E., Ozer, J., et Ruddick, K.G. (1996), Presentation of a family of turbulence closure models for stratified shallow water flows and preliminary application to the Rhine outflow region. Cont. Shelf. Res., 16, 101-130.

Luyten, P. et De Mulder, T., A module representing surface fluxes of momentum and heat. MUMM's contribution to MAST-0050-C. Technical report 9. Management Unit of the Mathematical Models of the North Sea and Scheldt Estuary, 1992.

Reynaud, T., Legrand, P., Mercier, H., et Barnier, B. (1998), A new analysis of hydrographic data in the Atlantic and its application to an inverse modelling study. Int. WOCoastal Engineering Newsletter, 32, 29-31.

Soulsby, R. (1997), Dynamics of marine sands, a manual for practical applications. London, Thomas Telford Publications. $256 \mathrm{p}$.

Tessier, C., Le Hir, P., et Lurton, X. (2006), Estimation de la matière en suspension à partir de l'intensité rétrodiffusée des courantomètres acoustiques à effet Doppler (ADCP). Comptes Rendus Geosciences, soumis. 